\title{
Developing and Implementing a Framework for System Level Measures: lessons from New Zealand
}

\author{
F Doolan-Noble, M Lyndon, A Hill, J Gray and R Gauld
}

\begin{abstract}
Background: Measuring performance is now the norm in health systems. System Level Measures (SLMs), implemented at New Zealand's Counties Manukau Health (CMH) are designed to support quality improvement activities undertaken across the health system using only a small set of measures. While the healthcare and performance measurement literature contains information regarding the facilitators and barriers to quality improvement initiatives, there is an absence of studies into whether these factors are germane to the establishment and implementation of a SLM framework.

Methods: A purposive sample of thirteen senior managers and clinicians involved in the construction and implementation of SLMs were invited to participate. Semi-structured telephone interviews were completed and recordings transcribed verbatim. Transcriptions were thematically analysed using a general inductive approach.
\end{abstract}

Findings: In total, ten interviews took place. Six facilitative themes were identified including: dispersed and focused leadership; communication; data; alignment of the measures with organisational strategic data; alignment of the measures with organisational strategic plans and values; stakeholder engagement; and a dedicated project team. Conversely, five themes were identified that hindered the process. These were: reaching consensus; perfection versus pragmatism; duplication and process burden; achieving buy-in and workload.

Discussion: The factors that facilitate and hinder establishing and implementing a framework of SLMs are common to other quality improvement approaches. However, this study demonstrated that these factors were also germane to SLMs. These findings are of particular relevance as researchers and policy makers elsewhere increasingly aim to adopt measurement arrangements for health systems that address equity, safety, quality, access and cost.

Abbreviations: $\mathrm{CMH}$ - Counties Manukau Health; DHB - District Health Board; IHI - Institute for Healthcare Improvement; QI - Quality Improvement; SLM - System Level Measure.

Key words: health systems; quality improvement; system level measures.

\section{Fiona Doolan-Noble}

Preventive and Social Medicine

Dunedin School of Medicine

University of Otago

Dunedin, New Zealand.

\section{Mataroria Lyndon}

Counties Manukau Health

Auckland, New Zealand.

\section{Andrew G Hill}

Counties Manukau Health

Auckland, New Zealand.

\section{J Gray}

Counties Manukau Health

Auckland, New Zealand.

\section{R Gauld}

Preventive and Social Medicince

University of Otago

Dunedin, New Zealand.

Correspondence:

fiona.doolan-noble@otago.ac.nz 


\section{Background}

The context for most healthcare systems in many developed countries is one of fiscal constraint but also of improving service integration, quality and performance. This article discusses the practical experience of developing and implementing a multidimensional framework of System Level Measures (SLMs), designed to support quality improvement within the context of a district-wide health system in New Zealand. By providing a description of factors that assisted and hampered the development of the framework, the article fills an important gap in relation to the use of SLMs in healthcare. Currently, there is only a limited body of SLMs related healthcare literature published, consisting of a report outlining the design principles behind SLMs, [1] and a paper examining the process of their development and implementation. [2] However, a report by Hibbert and colleagues does provide some useful information regarding the use of performance indicators within local health systems, albeit with a focus that is not specific to SLMs or their development. [3]

SLMs implemented at New Zealand's Counties Manukau Health (CM Health, a public hospital and health services provider, described in more detail below) are based on a framework developed by the Institute for Healthcare Improvement (IHI). [1] SLMs are intended to assist organisations to monitor their own improvement efforts towards achieving the IHI Triple Aim of improved service quality, with a focus on population health and consequent reduced healthcare spend. [4] In the case of CM Health the SLMs also align with their six 'executable strategies' (better health outcomes for all; first do no harm; system integration; ensuring financial sustainability; enabling high performing people; delivering patient and whānau centred care ${ }^{A}$ ) that are designed to support their journey towards achieving the IHI's Triple Aim. [4] SLMs provide data that:

- Demonstrate the longitudinal performance of the system;

- Enable the organisation to see how it is performing in relation to strategic plans for improvement;

- Facilitate comparisons with similar organisations; and

- Inform quality improvement planning. [1]

In theory, SLMs comprise a small set of measures [1] that bridge traditional intra and inter-organisational boundaries and support quality improvement to take place within

\footnotetext{
A Whānau centred care refers to care that is grounded in Māori culture and takes a holistic approach to improving the wellbeing of whānau (families) and addressing the needs of individuals within that whānau.
}

the global context of a health system. [5] While SLMs also support performance management they differ due to a focus on measuring the performance of a whole system and the contribution of its various parts, including hospitals and primary care services, to the overall performance of that system. Performance measurement, in contrast, focuses on performance within a single organisation, such as a hospital. [6] In addition, SLMs are recognised as supporting integration within health systems and progressing health reform toward integration over time. [3] This is potentially because the contributory measures that inform the SLMs relate to different parts of the system. Moreover, it is conceivable that the use of SLMs encourages everyone to become involved in quality improvement $(\mathrm{QI})$, as contributory measures are designed to measure activity that those at the frontline of clinical and service delivery consider relevant and which they can influence.

Limited health service-specific research has been undertaken to untangle factors that enable or constrain the development and implementation of a SLM framework. Kolberg and Elg identified four key challenges specific to developing performance measurement systems: reaching consensus around the measures to be used; maintaining competence in a wide range of fields within the project team; accepting scrutiny and critique of the project; and clarifying the end users of the system and determining their varying needs. [7] Additional barriers cited in the literature include a lack of dedicated human resources with the suitable skills to identify the appropriate measures and their related true drivers; the inflexible nature of information systems which, in healthcare, are frequently designed to enable the collection of administrative and clinical data and not necessarily constructed to report on performance measures; a focus on perfection which can stymie success, as can lack of staff engagement; and misjudging the time and expense required for development. $[6,8,9]$ Whilst there are clearly challenges associated with the development of such measures, there are also some recognised enablers. Leadership [9] and leadership distributed across the different levels of an organisation appear beneficial. [10] Acceptance of measurement throughout an organisation and the mapping of measures to an organisation's strategic objectives and its priorities and values are also recognised as enablers. $[6,8]$

Dixon-Woods and colleagues highlight factors that can impact negatively on the sustainability of Ql initiatives. [9] These factors include treating QI initiatives like a project with a beginning and an end, meaning the need to embed 
processes is limited and even missed; over-reliance on certain individuals; underestimating the need to be explicit about the intent of the measurement intervention and failing to demonstrate the relevancy of the QI activity. [9]

\section{Study setting}

In New Zealand, there are twenty publically funded District Health Boards (DHBs) created in 2000 by the Public Health and Disabilities Act. [11] The DHBs are each responsible for funding and providing public hospital and other healthcare services for a geographically-based population, including primary care and disability support services. CM Health is one of three DHBs in the most populous region of New Zealand, the Auckland metropolitan area, and services a population of approximately 500,000 people. The CM Health population is characterised by its youthfulness, high numbers of Māori, Pacific and Asian peoples and by high rates of deprivation. [12] In common with other DHBs in New Zealand, and with international trends, CM Health also has an ageing population and increasing rates of chronic illness. Consequently, as a funder and service provider, CM Health faces multiple challenges driven by its population's profile.
[12] These challenges and the focus on building a cohesive district health system underpinned the need to have a system of measures in place to determine the performance of the healthcare system, as well as opportunities for improvement. In addition, the aspirational goal set by the CEO of 'being as good as or better than comparable health systems anywhere in the world and beginning with being the best healthcare system in Australasia by December 2015', also required the establishment of a measurement framework.

To this end, CM Health commenced a phased process of developing a set of SLMs late in 2013 when a team was established to facilitate their development and implementation. The team comprised the following roles: SLM champions who were senior leaders who advocated for the incorporation of the SLMs into the health system; SLM coordinators who facilitated the compilation of the drill downs into the contributory measures (measures that influence a SLM) by managing the flow of communication and data required to complete a drill down (a drill-down is a report containing data which presents an organisation's

Figure 1: CM Health System Level Measures (System Level Measures are in dark blue ovals)

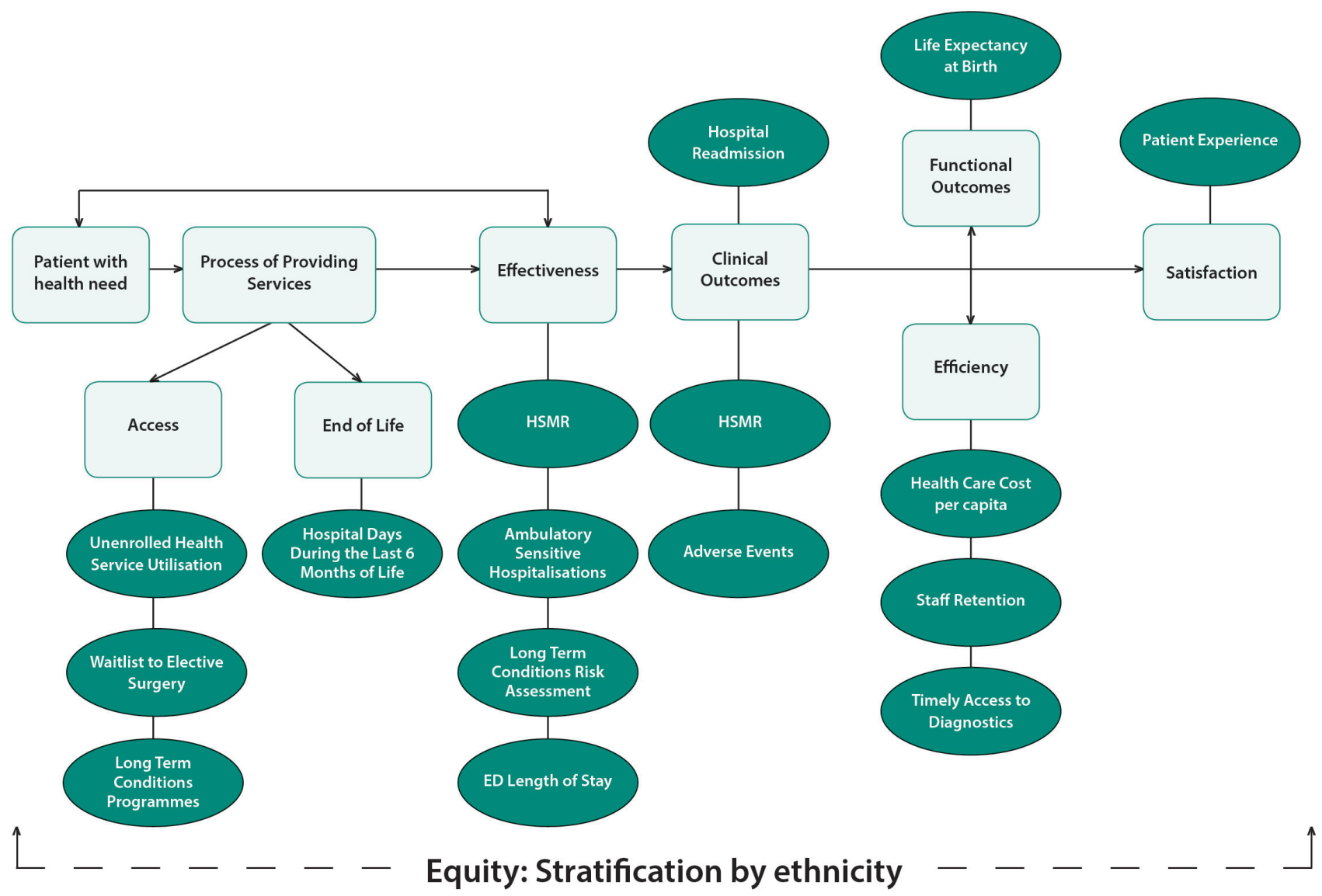

Adapted from the Institute of Healthcare Improvement Whole of Systems Measures. [1] 
performance for a selected SLM); a data analyst who had access to the data warehouse and analysed and presented the data in the drill downs and a quality improvement advisor who was an expert in analysing data and interpreting special cause variation to assist with the correct interpretation of the data. In addition, there was a SLMs Advisory Group comprising the head of Health Intelligence, Director of Allied Health and the two SLMs coordinators.

Some of the final group of SLMs agreed to by CM Health were pre-existing measures, although not previously used in the context of whole of system measurement, while other measures were agreed upon specifically for the SLM framework. The end result, finalised in 2014, was a suite of 16 SLMs (figure 1) that conceptually are similar to those used in other healthcare organisations, such as Sweden's Jönköping. [13]

This suite of measures is now live and is providing CM Health with a lens on quality of care, access, efficiency and health equity. Furthermore, SLMs are now influencing wider health policy within New Zealand.

\section{Methods}

As our aim was to understand the experiences of those involved with the development and implementation of the SLMs, a qualitative approach using semi-structured interviews was adopted. [14] A purposive sample [15] of thirteen senior managers and clinicians involved in the construction of the suite of SLMs was identified. All had involvement in the work either through their role on the developmental group, or in their capacity within the Clinical Governance Group or the Executive Leadership Team of CM Health. Invitees represented the spectrum of services provided by CM Health: population health, and primary and secondary care.
Participants were emailed an information sheet and consent form. [16] An interview schedule was developed to guide the semi-structured interviews; [14] these took place after the SLMs were implemented. All interviews were undertaken by one of the authors for consistency, recorded digitally and transcribed verbatim. Transcriptions were read by two of the authors and thematically analysed using a general inductive approach, [17] as the aim of the analysis was to determine if themes were evident in the interview data, not to answer an a priori question, as this is a relatively unexplored area in health services literature. The research protocol for this study was reviewed and approved by the University of Otago Human Research Ethics Committee, reference number D14/314.

\section{Findings}

Of the thirteen personnel invited to participate, two declined and one failed to respond. Analysis of the narratives revealed key factors that facilitated the development and implementation of the SLMs framework, as well as challenges to be negotiated. These are illustrated in Table 1.

\section{Facilitators}

\section{Leadership}

The leadership shown by the CEO was identified as vital. Interviewees felt he made tangible the aspirations of those working in the organisation when he set the goal of being the best healthcare system in Australasia by December 2015. By articulating this goal, he gave impetus to the need to establish a framework for measuring system performance and improvement. In addition, interviewees valued the leadership shown by the leader of the SLM initiative, 'Absolutely the right person to go forward with it (the initiative)'. The engagement of one of the clinical leaders within the organisation to work alongside the project team was viewed

Table 1: Factors that enable and constrain the development of SLMs

\begin{tabular}{|c|c|}
\hline ENABLING FACTORS & RESTRAINING FACTORS \\
\hline Leadership, including distributed leadership & Reaching consensus \\
\hline Communication & Perfection v pragmatism \\
\hline Data & Duplication and process burden \\
\hline Alignment and ownership & Buy-in \\
\hline Stakeholder engagement & \\
\hline Project team & \\
\hline
\end{tabular}


positively, 'I think it was really good having XX as the Clinical Champion because he could open lots of doors because of his clinical reputation.' Having the guidance of a senior manager who had established a similar system elsewhere was also judged beneficial. The distributed leadership provided, therefore, gave direction strategically, theoretically, clinically, experientially and from a project management perspective.

\section{Communication}

All interviewees were very clear about the rationale for establishing the SLM framework. This was viewed as enabling various activities underpinning improvement, such as benchmarking, as opposed to judgement, which can be counterproductive. They also considered it provided a mechanism for assessing progress towards the IHI Triple Aim [4] which the organisation uses to guide its planning process. Participants believed the SLMs assisted in monitoring progress towards the goal set by the CEO and could potentially facilitate comparisons with other health systems, nationally and internationally.

\section{Data}

The routinely collected data, which were repackaged to inform the reporting on SLMs, were seen as a key facilitator and viewed as a 'can-opener'. Interviewees spoke of the data, 'prompting conversations and debates that otherwise would not have occurred'; 'forcing you to look at the whole system' and 'making sense of the multiplicity of activities that take place within a health system. The data that underpin the SLMs and form the contributory measures were also deemed significant:

'After defining some system level measures actually building the conversations around the contributory measure we sparked really important discussions. It starts to drive at what the logic is behind our measurement and our improvement.'

\section{Alignment and ownership}

The importance of the SLMs aligning with CM Health's six executable strategies was also articulated, as a facilitator, as was having ownership of the measures. The latter was considered important as it enabled the organisation to, 'identify our own priorities and our own opportunities for improvement', as well as providing 'the ability to reflect on ourselves.' In New Zealand's government-funded health system this was considered preferable to the many measurement demands predetermined by the Ministry of Health. [18]

\section{Stakeholder engagement}

The project team considered it important to have broad stakeholder engagement: for example, 'we worked across different teams to enable them to propose measures'. They reported back on proposed measures and contributory measures and presented the various teams with information regarding how their proposed measures functioned. This level of engagement was seen as not only facilitating the development of a robust framework but also assisting with the implementation and utilisation of the framework once it became active. One interviewee summed up the engagement process as follows: 'You'd have to say it was a successful engagement process as the whole thing has been implemented.' However, the project team acknowledged that the effort required to engage with a range stakeholders was considerable,.. . there is a lot of hard work, the engagement stuff, a lot of hard work.' This in part was driven by the need to expand their stakeholder consultation due to the interest shown by many people in having input into the initiative.

\section{Project team}

The final facilitator acknowledged by interviewees was the presence of a dedicated project team. Interviewees recognised the initiative required a huge effort by the team, 'That puts a lot of work on the system level measure people as opposed to anybody else doing any of the work'.

\section{Challenges}

\section{Reaching consensus}

Certain factors were identified as hampering the establishment and implementation of the SLMs framework. At the development stage, reaching consensus was a cause of tension:

'There was a lot of appropriate fighting over inclusion and exclusion.' As a result, the initial plan to have twelve SLMs expanded to sixteen because 'there was the argument that we were not representing primary care enough'.

However, one interviewee summed it up:

'Well the biggest problem's been people having their own agenda. They're not really understanding what they're all about. So if people sort of think we need the primary care measure they're not really understanding what the point of the big dot is. Primary care is plainly a feature of the organisation, but you know, aspects of it are just feeders to a big dot. Umm, you know nothing in an organisation should really exist in isolation.'

\section{Perfection versus pragmatism}

Friction arose around the desire to establish a perfect set of measures and contributory measures versus taking a pragmatic, 'this is good enough' approach, as described by one interviewee:

'The huge challenge that came through all the time was a desire to make these perfect before we engaged in any 
further exploration and I know that um, I pushed very, very hard to get things on the table even if they weren't perfect'.

Many of those engaged with the development of the measures were accepting of this approach as it allowed for an area of interest to be acknowledged, for example, patient experience of care, even if appropriate data was lacking, therefore, limiting the usefulness of the measure in the interim.

\section{Duplication}

Duplication was another hurdle. Some measures already existed on other performance monitoring dashboards. Participants pointed out that the timetables of the different reporting requirements frequently did not align, resulting in process burden as another set of reports had to be generated and another set of analysis undertaken. The need to generate different reports for the same measures was driven by variations in definitions, denominators and numerators dependent on who the report was for.

\section{Buy-in}

Achieving buy-in was another challenge, 'that we probably got wrong initially'. Those involved in the stakeholder engagement felt that there was an initial underestimation of the number of people who wanted to be involved. Consequently, a wider engagement approach was instigated.

\section{Workload}

The final challenge was the workload associated with the SLM development and subsequent implementation. One participant pointed out that the work involved more than just determining, developing and assessing the appropriateness of a series of measures. It also included communication, reporting and associated work, such as, data analysis. Due to the newness of this approach, roles and responsibilities were perhaps not clearly defined, leaving the project team unsure about the parameters of their work:

'I said maybe we aren't able to do that, maybe our audience are the executives and boards and clinical governance and maybe it is for them to push it through, you know'.

The formative stage of the process including the need to have a project plan documented, appropriate resourcing estimations carried out and a business owner identified for the initiative was recognised as a key area for improvement.

\section{Discussion}

Several factors that enhanced but also hindered the development and implementation of SLMs within a health system were identified by participants in this study. Many of these factors have been reported elsewhere in the healthcare and performance management literature in relation to QI. [19-26] This study, however, illustrates their relevance in the context of SLM development and implementation. Leadership, not just that of the CEO was seen as crucial to the development and implementation process associated with the SLMs. The goal set by the CEO was seen as prioritising QI initiatives and spurring senior management to tackle the tasks ahead. In turn, the distributed model of leadership associated with the SLMs provided direction, promoted alignment and fostered commitment, factors recognised as key for improvement. $[27,28]$ The broader New Zealand health policy emphasis on leadership may have assisted CM Health, in that government, since 2009, has worked to support leadership development especially amongst health professionals. [29] The impact of this has varied amongst the $20 \mathrm{DHBs}$. [30] A key difference in the case of CM Health may well be a focus on developing leadership across the organisation and, in particular, on gaining broad commitment to the SLMs developmental process amongst managerial and clinical staff.

Data were both an enabler and an obstacle. Data were viewed as initiating conversations or, as one interviewee phrased it, the 'can-opener' during the developmental phase. As a result, the conversations and debate prompted questions, enhanced the understanding of the system as a whole, and altered the way people assessed problems. While the data, and the discussions and debates generated, were viewed as pivotal to the development of SLMs, processing and interpreting the data were viewed as resulting in duplication and, as such, producing an increased burden on some staff. This had the potential to derail the SLM development process. Arguably, the cross-organisational leadership and buy-in to SLMs created a momentum that countered this possibility.

Overall the narratives revealed a sense that the health system had been 'unlocked' by the provision of the SLMs and their contributory measures, resulting in a greater awareness of how the system that is CM Health functioned as a whole which, of course, is an implicit aim of whole system measurement. In other words, the data and discussions that took place throughout the development of the SLMs framework appeared to enable those involved with the initiative to 'make sense' of their health system. It helped them develop a shared understanding of how different components within the structures that underpin activity within the organisation all interrelate. Furthermore, the conversations promoted collaborative cross system thinking, as opposed to thinking in competitive service delivery silos. 
Thus, conversations, recognised as pivotal to 'sensemaking', [31] were integral to the process of developing the SLMs by engendering a greater appreciation of how the components of the system interconnect.

When asked about the benefits of the SLMs many interviewees spoke of the advantage of the measures being owned by the organisation, instead of being externally imposed which can lead to various levels of gaming and goal displacement. [32] Interviewees saw the SLMs as providing opportunities for reflection, generating a sense of accountability, and providing a sense of relevance to the organisation.

The use of a project team comprised of individuals who were highly regarded by the various stakeholders to generate buy-in and commitment was another enabler identified, with functional similarities to the 'knowledge broker' role reported elsewhere. [33] Interviewees noted the importance of making the time for project leaders to work on engaging and involving different teams in the developmental process, depending on the SLM in question. Critical to this was the role of clinical leadership, which, as noted was pivotal to building legitimacy of the project amongst front-line practising health professionals. Conversely, the time required for stakeholder engagement was a key challenge, as is often the case with initiatives that are additional to healthcare delivery, which is the primary focus for health professionals.

Essentially, the development of SLMs as a contribution to improvement efforts takes time: time for stakeholder engagement; time to debate the suitability and relevancy of various performance measures; time to determine the true drivers of performance measures (the contributory measures); and time to undertake analyses and develop reports. In addition, knowing who to engage with internally within the organisation was identified as a problem, partly driven by the uniqueness of the initiative and hence a level of unfamiliarity regarding who to engage with. Having a team to manage not only stakeholder engagement but all the other associated tasks was considered important by those interviewed, as found in other studies of cross organisational initiatives. [33-35]

Reaching consensus on the SLMs was recognised by several interviewees as a difficult process, causing tension and frustration. This was partly driven by confusion regarding the nature and functions of SLMs and the desire by various participants for the measures to reflect their specific area, as opposed to the broader health system. The desire for a perfect set of measures and ancillary contributory measures versus the desire to action the measures and modify them as issues emerged was an additional cause of friction.

The workload associated with the initiative was viewed as challenging by those intimately involved in its day-to-day facilitation. It appeared that the scope of the work and the changing skill set required, as the initiative evolved was not fully recognised at the outset. Consequently, the initiative leader was required to undertake functions which would normally be part of the role of other contributors meaning there was some propensity toward work intensification, shown elsewhere to be associated with improvement activities. [36]

The limitations of this study need acknowledgement. First, although interviewees spanned clinical and managerial roles, no one specifically representing population health at CM Health participated in the study. While a population health perspective is not necessarily the exclusive domain of the public health specialists, a population health perspective on the SLMs chosen and the process undertaken, which might differ from the views expressed by the clinicians and managers, is missing. Similarly, no one from the health intelligence and informatics team was interviewed, meaning the challenges described in this study related to data extraction and analyses are possibly understated. Second, as with any qualitative study, the data reported here are reflective of a small number of interviewees. [37] While saturation was reached in the interview process and there is no reason to believe any interviewee misrepresented the reality, there are potentially restrictions on the extent to which the findings could be translatable into other settings. [38] Third, three of this article's authors (ML, JG and AH) also participated as interviewees. While it could be considered that there is an element of conflict of interest in this, as noted, all interviews and thematic analyses of interview data were undertaken by two of the authors (FD-N and RG) with all transcripts and interviewees anonymised. The findings were discussed with the interviewee authors who provided assistance with interpretations. Final analytical and editorial decisions on material and discussions in this article rested with FD-N and RG.

SLMs are going to be developed by all of New Zealand's DHBs, [18] yet, as noted in this article, there remain challenges with implementing the approach. Set within the context of a New Zealand DHB, this study has identified factors that enable and hinder the development and establishment of a framework of SLMs. These findings are particularly relevant as researchers and policy makers elsewhere increasingly aim to adopt measurement arrangements for health systems 
that address equity, safety, quality, access and cost. [39] Very importantly, this study revealed the importance of a coming together of two streams of activity which, in the CM Health context, were pivotal to successful SLM development: the technical element of designing the measures and their contributory measures; and the leadership and organisational components required to ensure their establishment and implementation.

\section{Competing interests}

The authors declare that they have no competing interests.

\section{References}

1. Martin L, Nelson E, Lloyd R, Nolan T. Whole of System Measures. IHI Innovation Series white paper. Cambridge, Massachuetts: Institute for Healthcare Improvement; 2007.

2. Doolan-Noble F, Lyndon M, Hau S, Hill A, Gray J, Gauld R. How well does your healthcare system perform? Tracking progress towards the triple aim using system level measures. N Z Med J. 2015; 128(1415):44-50.

3. Hibbert P, Hannaford N, Long J, Plumb J, Braithwaite J. Final report: Performance indicators used internationally to report publicly on healthcare organisations and local health systems. Sydney: Australian Insititute of Health Innovation, University of New South Wales; 2013.

4. Berwick D, Nolan T, Whittington J. The triple aim: care, health and cost. Health Aff. 2008;27(3):759-69.

5. Bodenheimer T, Bojestig M, Henriks G. Making systemwide improvements in healthcare: lessons from Jonkoping County, Sweden. Qual Manag Healthcare. 2007;16(1):10-5.

6. Bourne M, Neely A, Platts K, Mills J. The success and failure of performance measurement initiatives. International Journal of Operations and Production Management. 2002;22(11):1288-310.

7. Kollberg B, Elg M. Challenges exerpienced in the development of performance measurement systems in Swedish healthcare. Qual Manag Healthcare. 2006;15(4):244-56.

8. Kennerley M, Neely A. A framework of the factors affecting the evolution of performance measurement systems. International Journal of Operations and Production Management. 2002; 22(11):1222-45.

9. Dixon-Woods M, McNicol S, Martin G. Ten challenges in improving quality in healthcare: lessons from the Health Foundation's programme evaluations and relevant literature. BMJ Qual Saf Online. 2012.

10. Allcock C, Dormon F, Taunt R, Dixon J. Constructive comfort: accelerating change in the NHS. London: The Health Foundation; 2015.

11. New Zealand Public Health and Disability Act, Stat. 91 (2000).

12. Counties Manukau District Health Board. About Counties Manukau District Health Board. Available from: http://www.counties manukau.health.nz/about_cmdhb/overview/printpopulation profile.pdf.

13. BC Patient Safety and Quality Council. Measurement strategies for improving the quality of care: A review of best practice. Vancouver (BC): BC Patient Safety and Quality Council; 2010.

14. Bryman A. Interviewing in qualitative research. Social Research Methods. 4th ed. Oxford: Oxford University Press; 2012.

15. Bryman A. Sampling in qualitative research. In: Social Research Methods. Oxford: Oxford University Press; 2012.
16. Opdenakker R. Advantages and disadvantages of four interview techniques in qualitative research. Qualitative Social Research [Internet]. 2006;7(4). Available from: http://www.qualitativeresearch.net/index.php/fqs/article/view/175/391.

17. Thomas D. A general inductive approach for qualitative data analysis 2003. Available from: http://www.fmhs.auckland.ac.nz/ soph/centres/hrmas/_docs/Inductive2003.pdf.

18. Ministry of Health. Integrated Performance and Incentive Framework. Expert Advisory Group. Final Report 19th February 2014. Wellington: Ministry of Health; 2014.

19. Shipton $H, C$ A, West M, Dawson J. The impact of leadership and quality climate on hospital performance. Int J Qual Health Care. 2008;20(6):439-45.

20. Bisognano M. Patient-centered leadership: more than a score: focusing on the patient will improve both quality and patient satisfaction. Healthcare Executive. 2012;27(6):70, 2, 4.

21. The King's Fund. Leadership and Engagement for Improvement in the NHS. London: The King's Fund; 2012.

22. Thomas E. Improving teamwork in healthcare: current approaches and the path forward. BMJ Qual Saf. 2011;20:647-50.

23. Luxford K, Safran DG, Delbanco T. Promoting patient-centered care: a qualitative study of facilitators and barriers in healthcare organizations with a reputation for improving the patient experience. Int J Qual Health Care. 2011;23(5):510-5.

24. Ovretveit J, Andreen-Sachs M, Carlsson J, Gustafsson H, Hansson J, Keller $\mathrm{C}$, et al. Implementing organisation and management innovations in Swedish healthcare: lessons from a comparison of 12 cases. J Health Organ Manag. 2012;26(2):237-57.

25. Klassen A, Miller A, Anderson N, Shen J, Schiariti V, O'Donnell M. Performance measurement and improvement frameworks in health, education and social services systems: a systematic review. Int J Qual Health Care . 2010;22(1):44-69.

26. Johnsen $A$. What does 25 years of experience tell us about the state of performance measurement in public policy and management? Public Money and Management. 2005;25(1):9-17.

27. Gauld R, Horsburgh S, Brown J. The clinical governance development index: results from a New Zealand Study. BMJ Qual Saf. 2011;20(11):947-53.

28. Leape L, Berwick D, Clancy C. Transforming healthcare: a safety imperative. Qual Saf Health Care. 2009;18:424-8.

29. Ministerial Task Group on Clinical Leadership. In good hands: Transforming clinical governance in New Zealand Wellington: Ministerial Task Group on Clinical Leadership; 2009. Available from: https://www.beehive.govt.nz/sites/all/files/In\%20Good\%20 Hands\%20Report.pdf.

30. Gauld R, Horsburgh S. Measuring progress with clinical governance development in New Zealand: perceptions of senior doctors in 2010 and 2012. BMC Health Serv Res. 2014;14(547):1-7.

31. Weick K, Sutcliffe K, Obstfeld D. Organising the process of sensemaking. Organisational Science. 2005;16(4):409-21.

32. Hood C. Gaming in targetworld: The targets approach to managing British public services. Public Administration Review. 2006; 66(4):515-21.

33. Conklin J, Lusk E, Harris M, Stolee P. Knowledge brokers in a knowledge network: the case of Seniors Health Research Transfer Network knowledge brokers. Implementation Science. 2013;8:7.

34. Iaquinto B, Ison R, Faggian R. Creating communities of practice: scoping purposeful design. Journal of Knowledge Management. 2011;15(1):4-21. 
35. Evans $\mathrm{S}$, Scarbrough $\mathrm{H}$. Supporting knowledge translation through collaborative translational research initiatives: 'bridging'versus 'blurring' boundaryspanning approaches in the UK CLAHRC initiative. Soc Sci Med. 2014;106:119-27.

36. Delbridge R, Turnbull P, Wilkinson B. Pushing back the frontiers: management control and work intensification under JIT/TqM factory regimes. New Technology Work and Employment. 1992; (2):97-106.

37. Patton M. Qualitative evaluation and research methods. Newbury Park: Sage Publications; 1990.

38. Mays N, Pope C, Popay J. Systematically reviewing qualitative and quantitative evidence to inform management and policy-making in the health field. J Health Serv Res Policy. 2005;10Suppl1:6-20.

39. Blumenthal D, McGinnis JM. Measuring Vital Signs: an IOM report on core metrics for health and healthcare progress. JAMA. 2015; 313(19):1901-2. 